\title{
Synthesis of pentaoxaspiroalkanes and pentaoxocanes catalyzed by lanthanide compounds
}

\author{
Nataliya N. Makhmudiyarova,* Guzeliya M. Khatmullina, Rustem Sh. Rakhimov, \\ Askhat G. Ibragimov, and Usein M. Dzhemilev
}

Institute of Petrochemistry and Catalysis, Russian Academy of Sciences, 141 Prospekt Oktyabrya, 450075 Ufa, Russian Federation

E-mail: nataliya.mnn@gmail.com

DOI: https://doi.org/10.24820/ark.5550190.p009.565

\begin{abstract}
An efficient method for the synthesis of pentaoxaspiroalkanes and pentaoxocanes by cyclocondensation of 1,1-bis(hydroperoxy)cycloalkanes and 1,1-bis(hydroperoxy)alkanes with formaldehyde catalyzed by $\mathrm{Sm}\left(\mathrm{NO}_{3}\right)_{3} \cdot 6 \mathrm{H}_{2} \mathrm{O}$ has been developed.
\end{abstract}

Keywords: Lanthanide catalysis, cyclocondensation, 1,1-bis(hydroperoxy)cycloalkanes, 1,1-bis(hydroperoxy)alkanes, formaldehyde, pentaoxaspiroalkanes

\section{Introduction}

Organic peroxides belong to a broad and highly demanded class of compounds. ${ }^{1,2}$ Interest in the development of new methods for the synthesis of cyclic peroxides is due to their antimalarial activity. ${ }^{3,4}$ We have shown earlier that pentaoxocanes are used in the synthesis of $N$ aryltetraoxazaspiroalkanes. ${ }^{5}$ The nitrogen-containing cyclic peroxides are promising compounds with antimalarial activity. ${ }^{2,6}$ The best known methods for the synthesis of pentaoxocanes are the acid-catalyzed reaction of $\alpha$-alkoxyhydroperoxides with aliphatic aldehydes, ${ }^{7-9}$ the acidolysis of aryl/alkyl cycloalkene ozonides with chlorosulfonic acid, ${ }^{4,10-12}$ and the reaction of bissilylisochromane with aromatic aldehydes. ${ }^{12}$ Drawbacks of the known methods of pentaoxocane synthesis include the low $(5 \%)$ or moderate $(34 \%)$ yields and the several steps needed to obtain the desired products. 


\section{Results and Discussion}

The purpose of this work is to develop a catalytic method for the selective synthesis of new spirocoupled pentaoxocanes 1 in high yields. When starting on this problem, we assumed that if cyclocondensation of $\alpha, \omega$-SH acids with formaldehyde affords oxadithiacycloalkanes, ${ }^{13}$ then cyclocondensation of 1,1-bis(hydroperoxy)cycloalkanes 2 ( $\alpha, \omega$-OHacids) with formaldehyde would provide a synthesis of pentaoxocanes. It was shown by tentative experiments that this reaction in the absence of a catalyst does not give pentaoxaspiroalkanes $\mathbf{1}$, while the reaction of $\mathbf{2}$ with aldehydes in the presence of traditional acid catalysts such as sulfuric acid or $\mathrm{BF}_{3} \mathrm{Et}_{2} \mathrm{O}$ gives rise to 1,2,4,5-tetraoxanes. ${ }^{14}$ In relation to the reaction of 1,1-bis(hydroperoxy)cyclohexane $\mathbf{2 b}$ with formaldehyde, we found that in the presence of $\mathrm{Sm}\left(\mathrm{NO}_{3}\right)_{3} \cdot 6 \mathrm{H}_{2} \mathrm{O}(5 \mathrm{~mol} \%)$ as a catalyst, the reaction carried out at $\sim 20^{\circ} \mathrm{C}$ for $6 \mathrm{~h}$ in THF gives the pentaoxaspiroalkane $1 \mathrm{~b}$ in $95 \%$ yield. The $\mathrm{Sm}\left(\mathrm{NO}_{3}\right)_{3} \cdot 6 \mathrm{H}_{2} \mathrm{O}$ catalyst was chosen due to its successful use in our previous work to catalyze the cyclocondensation of NHacids with formaldehyde and $\alpha, \omega$-diols or $\alpha, \omega$-dithiols to afford 1,5,3dioxazepanes ${ }^{14}$ or 1,5,3-dithiazacycloalkanes. ${ }^{15-22}$
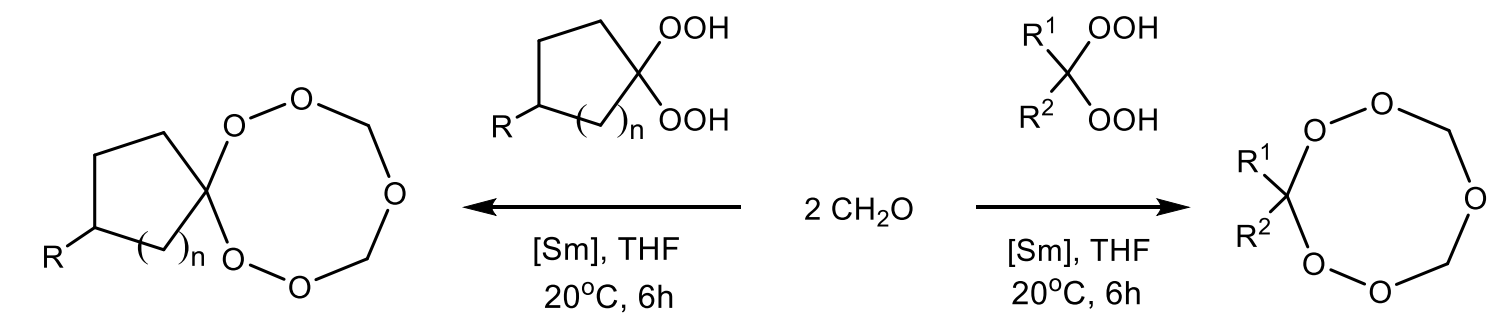

$$
\begin{gathered}
\mathrm{R}=\mathrm{H}, \mathrm{n}=1(\mathbf{1 a}), 2(\mathbf{1 b}), 3(\mathbf{1 d}), 4(\mathbf{1 e})[\mathrm{Sm}]=\mathrm{Sm}\left(\mathrm{NO}_{3}\right)_{3} \cdot 6 \mathrm{H}_{2} \mathrm{O} \\
\mathrm{R}=\mathrm{Me}, \mathrm{n}=2(\mathbf{1 c})
\end{gathered}
$$

$$
\begin{gathered}
\mathrm{R}^{1}=\mathrm{R}^{2}=\mathrm{Me}(\mathbf{3}, \mathbf{7}) \\
\mathrm{R}^{1}=\mathrm{Me}, \mathrm{R}^{2}=\mathrm{Bu}^{\mathrm{t}}(\mathbf{4 , 8}) \\
\mathrm{R}^{1}=\mathrm{R}^{2}=\mathrm{Bu}(\mathbf{5}, \mathbf{9}) \\
\mathrm{R}^{1}, \mathrm{R}^{2}=\text { adamantane-2,2-diyl }(\mathbf{6}, \mathbf{1 0})
\end{gathered}
$$

Scheme. The synthesis of pentaoxaspiroalkanes and pentaoxocanes by cyclocondensation of 1,1bis(hydroperoxy)cycloalkanes and 1,1-bis(hydroperoxy)alkanes with formaldehyde.

By NMR spectroscopic methods, compound $\mathbf{1 b}$ was identified as 7,8,10,12,13-pentaoxaspiro[5.7]tridecane based on signals at $109.98 \mathrm{ppm}$, typical of $\mathrm{sp}^{3}$-hybridized carbon bearing two oxygen functions, and the signal at $92.30 \mathrm{ppm}$, typical of carbons in the $-\mathrm{CH}_{2}-\mathrm{O}-\mathrm{CH}_{2}-$ system. The ${ }^{1} \mathrm{H}$ NMR spectrum exhibits a singlet at $5.17 \mathrm{ppm}$ due to the $-\mathrm{O}-\mathrm{CH}_{2}-\mathrm{O}-\mathrm{CH}_{2}-\mathrm{O}$ ring protons correlated in the HSQC experiment with the $\delta 92.30 \mathrm{ppm}$ carbon signal. The multiplets at 1.76, 1.54 , and $1.43 \mathrm{ppm}$ refer to the cyclohexane ring in $\mathbf{1 b}$. The structure of $7,8,10,12,13$ pentaoxaspiro[5.7]tridecane $\mathbf{1 b}$ was additionally confirmed by MALDI-TOF mass spectrometry. The spectrum contains a molecular ion fragment at $m / z 191[\mathrm{M}-\mathrm{H}]^{+}$, indicating the formation of compound $\mathbf{1 b}$ under the reaction conditions. 
It was shown by subsequent experiments that under the conditions selected $(5 \mathrm{~mol} \%$ $\left.\mathrm{Sm}\left(\mathrm{NO}_{3}\right)_{3} \cdot 6 \mathrm{H}_{2} \mathrm{O}, 20^{\circ} \mathrm{C}, 6 \mathrm{~h}\right)$, the yield of the target product $\mathbf{1 b}$ decreases in the following sequence of solvents: $\mathrm{THF}(95 \%)>\mathrm{CH}_{2} \mathrm{Cl}_{2}(85 \%)>\mathrm{Et}_{2} \mathrm{O}(79 \%)>\mathrm{C}_{6} \mathrm{H}_{12}(15 \%)>\operatorname{EtOAc}(10 \%)>\mathrm{C}_{2} \mathrm{H}_{5} \mathrm{OH}$ $(7 \%)$.

Apart from $\mathrm{Sm}\left(\mathrm{NO}_{3}\right)_{3} \cdot 6 \mathrm{H}_{2} \mathrm{O}$, we tested some other lanthanide (Ho, Tb, Dy, Nd, La) salts as catalysts in the cyclocondensation reaction. The reactions were conducted at $\sim 20^{\circ} \mathrm{C}$ in THF in the presence of $5 \mathrm{~mol}$. \% of catalyst. Under these conditions, selective formation of $\mathbf{1 b}$ took place in the following yields depending on the catalyst: $84 \%\left(\mathrm{Ho}\left(\mathrm{NO}_{3}\right)_{3} \cdot 5 \mathrm{H}_{2} \mathrm{O}\right)>72 \%\left(\mathrm{TbCl}_{3} \cdot 6 \mathrm{H}_{2} \mathrm{O}\right)>$ $67 \%\left(\mathrm{DyCl}_{3} \cdot 6 \mathrm{H}_{2} \mathrm{O}\right)>61 \%\left(\mathrm{NdCl}_{3}\right)>58 \%\left(\mathrm{La}\left(\mathrm{NO}_{3}\right)_{3}\right)$.

Under the optimal conditions for the preparation of $\mathbf{1 b}\left(5 \mathrm{~mol} . \%\right.$ of $\mathrm{Sm}\left(\mathrm{NO}_{3}\right)_{3} \cdot 6 \mathrm{H}_{2} \mathrm{O}$, THF, $20{ }^{\circ} \mathrm{C}, 6 \mathrm{~h}$ ), the cyclocondensation of 1,1-bis(hydroperoxy)cycloalkanes $\mathbf{2 a , c - e ^ { 1 4 }}$ with formaldehyde results in the selective formation of pentaoxaspiroalkanes 1a,c-e in yields of $90 \%$ (1a) $>85 \%($ 1c) $>71 \%$ (1d) $>69 \%$ (1e) (scheme).

To determine the possibility of selective synthesis of pentaoxocanes, we studied the $\mathrm{Sm}\left(\mathrm{NO}_{3}\right)_{3} \cdot 6 \mathrm{H}_{2} \mathrm{O}$ catalyzed reaction of 1,1-bis(hydroperoxy)alkanes 3-6 ${ }^{14}$ with formaldehyde. We found that this reaction provides an effective way to obtain pentaoxocanes. The yield of 1,2,4,5,7pentaoxocanes 7-10 decreases in the sequence 2,2-dihydroperoxypropane $3(98 \%)>5,5$ dihydroperoxynonane $5(63 \%)>$ 2,2-dihydroxyadamantane $6(53 \%)$ when using $5 \mathrm{~mol}$. \% of $\mathrm{Sm}\left(\mathrm{NO}_{3}\right)_{3} \cdot 6 \mathrm{H}_{2} \mathrm{O}$ in THF solution at $20{ }^{\circ} \mathrm{C}$ for $6 \mathrm{~h}$ (scheme). The reaction time for the synthesis of adamantane-2-spiro-3'-1', 2', $4^{\prime}, 5^{\prime}, 7^{\prime}$-pentaoxocane 10 was $10 \mathrm{~h}$.

\section{Conclusions}

We have developed a new selective method for the synthesis of pentaoxaspiroalkanes and pentaoxocanes by $\mathrm{Sm}\left(\mathrm{NO}_{3}\right)_{3} \cdot 6 \mathrm{H}_{2} \mathrm{O}$ catalyzed cyclocondensation of 1,1-bis(hydroperoxy)cycloalkanes and gem-bis(hydroperoxy)alkanes with formaldehyde.

\section{Experimental Section}

General. All reactions were performed at room temperature under an air atmosphere in a round bottom flask equipped with a magnetic stir bar. The ${ }^{1} \mathrm{H}$ and ${ }^{13} \mathrm{C}$ NMR spectra were recorded on a Bruker Avance-400 spectrometer (400 and $100 \mathrm{MHz}$, respectively) in $\mathrm{CDCl}_{3}$, internal standard was TMS. Two-dimensional homonuclear (COSY, NOESY) and heteronuclear (HSQC, HMBC) experiments were carried out under standard Bruker pulse sequences at the same operating frequencies. The mixing time for the NOESY experiments was $0.3 \mathrm{~s}$. Mass spectra were recorded on a Bruker Autoflex III MALDI TOF instrument with $\alpha$-cyano-4-hydroxycinnamic acid (CHCA) as a matrix. Samples of the compounds were prepared by the "dried droplet method". C/H analyses were carried out on a Carlo Erba 1108 analyzer, O analyses on a Carlo Erba 1106 analyzer. The 
progress of reactions was monitored by TLC on Sorbfil (PTSKh-AF-V) plates, visualization with $\mathrm{I}_{2}$ vapour.

The synthesis of the gem-bishydroperoxides 3-6 was as reported in the literature. ${ }^{10}$

Cyclocondensation of 1,1-bis(hydroperoxy)cycloalkanes and gem-bis(hydroperoxy)alkanes with formaldehyde catalyzed by $\mathrm{Sm}\left(\mathrm{NO}_{3}\right)_{3} \cdot \mathbf{6 H}_{2} \mathrm{O}$. General procedure A Schlenk vessel mounted on a magnetic stirrer was charged at $20^{\circ} \mathrm{C}$ with tetrahydrofuran $(5 \mathrm{ml})$, aqueous (37\%) formaldehyde $(1.46 \mathrm{ml}, 20 \mathrm{mmol})$, and the selected bis(hydroperoxy)cycloalkane [ $\mathrm{gem}$ bis(hydroperoxy)alkane] $(10 \mathrm{mmol})$. Then $\mathrm{Sm}\left(\mathrm{NO}_{3}\right)_{3} \cdot 6 \mathrm{H}_{2} \mathrm{O}(0.222 \mathrm{~g}, 5 \mathrm{~mol}$. \% relative to 1,1 bis(hydroperoxy)cycloalkane) was added. The reaction mixture was stirred at $\sim 20{ }^{\circ} \mathrm{C}$ for $6 \mathrm{~h}$ and tetrahydrofuran was evaporated. $\mathrm{Et}_{2} \mathrm{O}(10 \mathrm{ml})$ was added and the mixture was washed with water $(4 \times 5 \mathrm{ml})$. The ethereal layer was dried $\left(\mathrm{MgSO}_{4}\right)$ and concentrated to isolate pentaoxaspiroalkanes as oily liquids stable during storage at room temperature. Monitoring of the progress of reactions was effected by TLC, eluent was hexane : EtOAc, 5:1 (compounds 1a-f, 7-10), visualization with $\mathrm{I}_{2}$ vapor. The residue (compounds 1a-f, 7-10) was chromatographed on a column with $\mathrm{SiO}_{2}$ (eluent was hexane : EtOAc, 5:1) to isolate pure heterocyclic products.

6,7,9,11,12-Pentaoxaspiro[4.7]dodecane (1a). Colorless oil $(1.584 \mathrm{~g}, 90 \%), n_{D}^{20} 1.4564 .{ }^{1} \mathrm{H}$ NMR (DMSO- $\left.d_{6}, 400 \mathrm{MHz}\right) \delta$ 1.62-1.66 (m, 4H, $\left.\mathrm{H}_{2} \mathrm{C}\right), 1.86-1.89\left(\mathrm{~m}, 4 \mathrm{H}, \mathrm{H}_{2} \mathrm{C}\right), 5.04(\mathrm{~s}, 4 \mathrm{H}$, $\left.\mathrm{OH}_{2} \mathrm{CO}\right)$ ppm. ${ }^{13} \mathrm{C}$ NMR (DMSO- $\left.d_{6}, 100 \mathrm{MHz}\right) \delta 24.42\left(\mathrm{CH}_{2} \mathrm{CH}_{2}\right), 34.01\left(\mathrm{CH}_{2} \mathrm{CH}_{2}\right), 92.31$ $\left(\mathrm{OCH}_{2} \mathrm{O}\right), 119.90(\mathrm{C})$ ppm. MALDI TOF, $m / z$ : $175[\mathrm{M}-\mathrm{H}]^{+}$. Anal. Calcd. for $\mathrm{C}_{7} \mathrm{H}_{12} \mathrm{O} 5$ : C, 47.72; H, 6.87; O 45.41. Found: C, 47.67; H, 6.80; O, $44.35 \%$.

7,8,10,12,13-Pentaoxaspiro[5.7]tridecane (1b). Colorless oil $(1.805 \mathrm{~g}, 95 \%), n_{D}^{20}$ 1.5262. ${ }^{1} \mathrm{H}$ NMR (DMSO- $d_{6}, 400$ MHz) $\delta$ 1.43-1.44 (m, 4H, $\mathrm{H}_{2} \mathrm{C}$ ), 1.54-1.55 (m, 2H, $\left.\mathrm{H}_{2} \mathrm{C}\right), 1.76-1.83$ (m, $4 \mathrm{H}, \mathrm{H}_{2} \mathrm{C}$ ), 5.17 (s, 4H, OH${ }_{2} \mathrm{CO}$ ) ppm. ${ }^{13} \mathrm{C} \mathrm{NMR}$ (DMSO-d, $\left.100 \mathrm{MHz}\right) \delta 22.35\left(\mathrm{CH}_{2} \mathrm{CH}_{2}\right), 25.18$ $\left(\mathrm{CH}_{2}\right), 29.98\left(\mathrm{CH}_{2} \mathrm{CH}_{2}\right), 92.30\left(\mathrm{OCH}_{2} \mathrm{O}\right), 109.98(\mathrm{C}) \mathrm{ppm}$. MALDI TOF, $m / z: 189[\mathrm{M}-\mathrm{H}]^{+}$. Anal. Calcd. for $\mathrm{C}_{8} \mathrm{H}_{14} \mathrm{O} 5$ : C, 50.52; H, 7.42; O 42.06. Found: C, 50.48; H, 7.34; O, $42.00 \%$.

3-Methyl-7,8,10,12,13-pentaoxaspiro[5.7]tridecane (1c). Colorless oil $(1.734 \mathrm{~g}, 85 \%), n_{D}^{20}$ 1.4703. ${ }^{1} \mathrm{H}$ NMR (DMSO- $\left.d_{6}, 400 \mathrm{MHz}\right) \delta 0.88-0.89\left(\mathrm{~m}, 3 \mathrm{H}, \mathrm{CH}_{3}\right), 1.11-1.15(\mathrm{~m}, 1 \mathrm{H}, \mathrm{HC}), 1.40-$ $1.56\left(\mathrm{~m}, 6 \mathrm{H}, \mathrm{H}_{2} \mathrm{C}\right), 5.04\left(\mathrm{~s}, 4 \mathrm{H}, \mathrm{OH}_{2} \mathrm{CO}\right) \mathrm{ppm} .{ }^{13} \mathrm{C} \mathrm{NMR}\left(\mathrm{DMSO}-d_{6}, 100 \mathrm{MHz}\right) \delta 21.94\left(\mathrm{CH}_{3}\right)$, $30.17\left(\mathrm{CH}_{2} \mathrm{CH}_{2}\right), 30.75\left(\mathrm{CH}_{2} \mathrm{CH}_{2}\right), 31.55(\mathrm{CH}), 92.32\left(\mathrm{OCH}_{2} \mathrm{O}\right), 108.85(\mathrm{C}) \mathrm{ppm}$. MALDI TOF, $m / z: 203[\mathrm{M}-\mathrm{H}]^{+}$. Anal. Calcd. for $\mathrm{C}_{9} \mathrm{H}_{16} \mathrm{O} 5$ : C, 52.93; H, 7.90; O 39.17. Found: C, 52.87; H, 7.84; $\mathrm{O}, 39.09 \%$.

8,9,11,13,14-Pentaoxaspiro[6.7]tetradecane (1d). Colorless oil $(1.448 \mathrm{~g}, 71 \%), n_{D}^{20} 1.4591 .{ }^{1} \mathrm{H}$ NMR (DMSO-d $\left.d_{6} 400 \mathrm{MHz}\right) \delta 1.24-1.29\left(\mathrm{~m}, 4 \mathrm{H}, \mathrm{H}_{2} \mathrm{C}\right.$ ), 1.45-1.51 (m, 4H, $\mathrm{H}_{2} \mathrm{C}$ ), 1.75-1.82 (m, $2 \mathrm{H}, \mathrm{H}_{2} \mathrm{C}$ ), 2.31-2.34 (m, 4H, $\left.\mathrm{H}_{2} \mathrm{C}\right), 5.03$ (s, 4H, OH $2 \mathrm{CO}$ ) ppm. ${ }^{13} \mathrm{C} \mathrm{NMR}$ (DMSO-d, $\left.100 \mathrm{MHz}\right) \delta$ $24.15\left(\mathrm{CH}_{2} \mathrm{CH}_{2}\right), 30.14\left(\mathrm{CH}_{2} \mathrm{CH}_{2}\right), 43.75\left(\mathrm{CH}_{2} \mathrm{CH}_{2}\right), 92.08\left(\mathrm{OCH}_{2} \mathrm{O}\right), 113.90$ (C) ppm. MALDI TOF, $m / z: 203[\mathrm{M}-\mathrm{H}]^{+}$. Anal. Calcd. for $\mathrm{C}_{9} \mathrm{H}_{16} \mathrm{O}$ s: C, 52.93; H, 7.90; O 39.17. Found: C, 52.85; $\mathrm{H}, 7.86 ; \mathrm{O}, 39.11 \%$. 
1,2,4,6,7-Pentaoxaspiro[7.7]pentadecane (1e). Colorless oil $(1.504 \mathrm{~g}, 69 \%), n_{D}^{20} 1.4631 .{ }^{1} \mathrm{H}$ NMR (DMSO-d6, $400 \mathrm{MHz}) \delta$ 1.45-1.51 (m, 4H, $\left.\mathrm{H}_{2} \mathrm{C}\right), 1.77-1.79\left(\mathrm{~m}, 10 \mathrm{H}, \mathrm{H}_{2} \mathrm{C}\right), 5.01(\mathrm{~s}, 4 \mathrm{H}$, $\left.\mathrm{OH}_{2} \mathrm{CO}\right)$ ppm. ${ }^{13} \mathrm{C}$ NMR (DMSO- $\left.d_{6}, 100 \mathrm{MHz}\right) \delta 25.57\left(\mathrm{CH}_{2} \mathrm{CH}_{2}\right), 27.15\left(\mathrm{CH}_{2} \mathrm{CH}_{2} \mathrm{CH}_{2}\right), 41.48$ $\left(\mathrm{CH}_{2} \mathrm{CH}_{2}\right), 92.25\left(\mathrm{OCH}_{2} \mathrm{O}\right), 113.05(\mathrm{C}) \mathrm{ppm}$. MALDI TOF, $m / z: 217[\mathrm{M}-\mathrm{H}]^{+}$. Anal. Calcd. for $\mathrm{C}_{10} \mathrm{H}_{18} \mathrm{O}$ : $\mathrm{C}, 55.03$; H, 8.31; O 36.65. Found: C, 54.98; H, 8.28; O, $36.60 \%$.

3,3-Dimethyl-1,2,4,5,7-pentaoxocane (7). Colorless oil (1.471 g, 98\%), $n_{D}^{20}$ 1.4013. ${ }^{1} \mathrm{H}$ NMR $\left(\mathrm{DMSO}-d_{6}, 400 \mathrm{MHz}\right) \delta 1.25\left(\mathrm{c}, 6 \mathrm{H}, \mathrm{H}_{3} \mathrm{C}\right), 5.01\left(\mathrm{~s}, 4 \mathrm{H}, \mathrm{OH}_{2} \mathrm{CO}\right) \mathrm{ppm} .{ }^{13} \mathrm{C}$ NMR (DMSO- $d_{6}, 100$ MHz) $\delta 20.05\left(\mathrm{CH}_{3}\right), 92.04\left(\mathrm{OCH}_{2} \mathrm{O}\right), 109.03$ (C) ppm. MALDI TOF, $m / z: 149[\mathrm{M}-\mathrm{H}]^{+}$. Anal. Calcd. for $\mathrm{C}_{5} \mathrm{H}_{10} \mathrm{O} 5$ : C, 40.00; H, 6.71; O 53.29. Found: C, 39.95; H, 6.65; O, $53.25 \%$.

3-(t-Butyl)-3-methyl-1,2,4,5,7-pentaoxocane (8). Pale yellow oil (1.582 g, 82\%), $n_{D}^{20} 1.4742$. ${ }^{1} \mathrm{H}$ NMR (DMSO- $\left.d_{6}, 400 \mathrm{MHz}\right) \delta 1.05\left(\mathrm{c}, 12 \mathrm{H}, \mathrm{H}_{3} \mathrm{C}\right), 1.20\left(\mathrm{c}, 3 \mathrm{H}, \mathrm{H}_{3} \mathrm{C}\right.$ ), $5.03\left(\mathrm{~s}, 4 \mathrm{H}, \mathrm{OH}_{2} \mathrm{CO}\right)$ ppm. ${ }^{13} \mathrm{C}$ NMR (DMSO-d, $\left.100 \mathrm{MHz}\right) \delta 19.57\left(\mathrm{CH}_{3}\right), 22.15\left(\mathrm{CH}_{3}\right), 39.41(\mathrm{C}), 91.08\left(\mathrm{OCH}_{2} \mathrm{O}\right)$, 109.05 (C) ppm. MALDI TOF, m/z: $191[\mathrm{M} \mathrm{-} \mathrm{H}]^{+}$. Anal. Calcd. for $\mathrm{C}_{8} \mathrm{H}_{16} \mathrm{O} 5: \mathrm{C}, 49.99 ; \mathrm{H}, 8.39$; O 41.62. Found: C, 49.94; H, 8.35; O, $41.77 \%$.

3,3-Dibutyl-1,2,4,5,7-pentaoxocane (9). Colorless oil (1.474 g, 63\%), $n_{D}^{20}$ 1.4654. ${ }^{1} \mathrm{H}$ NMR (DMSO- $\left.d_{6}, 400 \mathrm{MHz}\right) \delta$ 0.97-1.01 (m, 6H, $\left.\mathrm{H}_{3} \mathrm{C}\right), 1.22-1.25\left(\mathrm{~m}, 8 \mathrm{H}, \mathrm{H}_{2} \mathrm{C}\right), 1.36-1.41\left(\mathrm{~m}, 4 \mathrm{H}, \mathrm{H}_{2} \mathrm{C}\right)$, $5.01\left(\mathrm{~s}, 4 \mathrm{H}, \mathrm{OH}_{2} \mathrm{CO}\right) \mathrm{ppm} .{ }^{13} \mathrm{C} \mathrm{NMR}$ (DMSO-d6, $\left.100 \mathrm{MHz}\right) \delta 19.45\left(\mathrm{CH}_{3}\right), 20.45\left(\mathrm{CH}_{2}\right), 23.76$ $\left(\mathrm{CH}_{2}\right), 25.32\left(\mathrm{CH}_{2}\right), 29.87\left(\mathrm{CH}_{2}\right), 91.02\left(\mathrm{OCH}_{2} \mathrm{O}\right), 109.52(\mathrm{C}) \mathrm{ppm}$. MALDI TOF, m/z: 233 [M $\mathrm{H}]^{+}$. Anal. Calcd. for $\mathrm{C}_{11} \mathrm{H}_{22} \mathrm{O} 5$ : C, 56.39; H, 9.46; O 34.14. Found: C, 56.34; H, 9.40; O, $34.30 \%$. Adamantane-2-spiro-3' $-\mathbf{1}^{\prime}, \mathbf{2}^{\prime}, \mathbf{4}^{\prime}, \mathbf{5}^{\prime}, \mathbf{7}^{\prime}$-pentaoxocane (10). Colorless oil (1.282 g, 53\%), $n_{D}^{20}$ 1.5241. ${ }^{1} \mathrm{H}$ NMR (DMSO- $\left.d_{6}, 400 \mathrm{MHz}\right) \delta 1.18-2.27$ (m, 14H, CH, $\mathrm{H}_{2} \mathrm{C}$ ), 5.09 (s, 4H, OH${ }_{2} \mathrm{CO}$ ). ${ }^{13} \mathrm{C}$ NMR (DMSO- $\left.d_{6}, 100 \mathrm{MHz}\right) \delta 29.74(\mathrm{CH}), 30.95\left(\mathrm{CH}_{2}\right), 33.57(\mathrm{CH}), 37.33\left(\mathrm{CH}_{2}\right), 91.07$ $\left(\mathrm{OCH}_{2} \mathrm{O}\right), 112.57(\mathrm{C})$. MALDI TOF, $m / z: 241[\mathrm{M}-\mathrm{H}]^{+}$. Anal. Calcd. for $\mathrm{C}_{12} \mathrm{H}_{18} \mathrm{O}$ : $\mathrm{C}, 59.49 ; \mathrm{H}$, 7.49; O 33.02. Found: C, 59.44; H, 7.42; O, $29.99 \%$.

\section{Acknowledgments}

This work was financially supported by the Russian Foundation for Basic Research, Russia (RFBR Grants 14-03-00240, 14-03097023, 16-2910687, SP-951.2015.4 and Scientific School 6651.2016.3).

\section{References and notes}

1. Jones, C.W. Application of Hydrogen Peroxides and Derivatives; Royal Society of Chemistry: Cambridge, 1999.

2. Organic Peroxides: Ando, W. Ed; Wiley: New York, 1992. 
3. Tang, Y.; Dong, Y.; Venerstrom, J. L. Med. Research Rev. 2004, 24, 425-448. http://dx.doi.org/10.1002/med.10066

4. .Opsenica D.M.; Šolaja B.A. J. Serb. Chem. Soc. 2009, 74, 1155-1193. http://dx.doi.org/10.2298/JSC0911155O

5. Makhmudiyarova, N. N.; Khatmullina, G. M.; Rakhimov, R. Sh.; Meshcheryakova, E. S.; Ibragimov, A. G.; Dzhemilev, U. M. Tetrahedron, 2016, 72, 3277-3281. http://dx.doi.org/10.1016/j.tet.2016.04.055

6. Opsenica, D. M.; Šolaja B. A. Macedonian Journal of Chemistry and Chemical Engineering 2012, 31, 137-182. http://www.mjcce.org.mk/index.php/MJCCE/article/view/50/50

7. McCullough, K. J.; Ushigoe, Y.; Tanaka, S.; Kawamura, S.; Masuyama, A.; Masatomo N. J. Chem. Soc., Perkin Trans. 1, 1998, 3059-3064. http://dx.doi.org/10.1039/a804605k

8. Ushigoe, Y.; Nojima, M.; McCullough, K. J. Chem. Lett. 1995, 8, 705-706. http://dx.doi.org/10.1246/cl.1995.705

9. Ushigoe, Y.; Tanaka, S.; Nojima, M.; McCullough, K. J. Tetrahedron Lett. 1994, 35, 97419744. http://dx.doi.org/10.1016/0040-4039(94)88374-2

10. Miura, M.; Nojima, M. J. Am. Chem. Soc. 1980, 102, 288-291. http://dx.doi.org/10.1021/ja00521a045

11. Miura, M.; Nojima, M.; Kusabayashi, S.; Nagaze, S. J. Am. Chem. Soc. 1983, 103, 1789-1796. http://dx.doi.org/10.1021/ja00397a034

12. Kim, H.-S.; Tsuchiya, K.; Shibata, Y.; Wataya, Y.; Ushigoe, Y.; Masuyama, A.; Nojima, M.; McCullough, K. J. J. Chem. Soc., Perkin Trans. 1, 1999, 1867-1870. http://dx.doi.org/10.1039/a900826h

13. Murzakova, N.N.; Prokof'ev, K.I.; Tyumkina, T.V.; Ibragimov, A.G. Russ. J. Org. Chem. 2012, 48, 588-593. http://dx.doi.org/10.1134/S1070428012040215

14. Terent'ev, A. O.; Platonov, M. M.; Ogibin Y. N.; Nikishin, G. I. Synth. Comm. 2007, 37, 12381287. http://dx.doi.org/10.1080/00397910701226384

15. Makhmudiyarova, N. N.; Prokof'ev, K. I.; Mudarisova, L. V.; Ibragimov, A. G.; Dzhemilev, U. M. Russ. J. Org. Chem. 2013, 49, 750-753. http://dx.doi.org/10.1134/S1070428013050217

16. Makhmudiyarova, N. N.; Prokof’ev, K. I.; Mudarisova, L. V.; Ibragimov, A. G.; Dzhemilev, U. M. Russ. J. Org. Chem. 2013, 49, 655-657. http://dx.doi.org/10.1134/S1070428013050023

17. Makhmudiyarova, N. N.; Prokof'ev, K. I.; Mudarisova, L. V.; Ibragimov, A. G.; Dzhemilev, U. M. Russ. J. Org. Chem. 2013, 49, 658-662. http://dx.doi.org/10.1134/S1070428013050035 
18. Rakhimova, E. B.; Ismagilov, R. A.; Zainullin, R. A.; Ibragimov, A. G.; Dzhemilev, U. M. Chem. Heterocycl. Compd. 2013, 49, 1237-1242.

http://dx.doi.org/10.1007/s10593-013-1368-0

19. Rakhimova, E. B.; Ismagilov, R. A.; Zainullin, R. A.; Galimzyanova, N. F.; Ibragimov. A. G. Russ. J. Appl. Chem. 2013, 86, 1504-1508.

http://dx.doi.org/10.1134/S1070427213100066

20. Khairullina, R. R.; Akmanov, B. F.; Kunakova, R. V.; Ibragimov, A. G.; Dzhemilev, U. M. Russ. Chem. Bull. Int. Edit. 2013, 62, 98-103.

http://dx.doi.org/10.1007/s11172-013-0013-5

21. Khairullina, R. R.; Akmanov, B. F.; Starikova, Z. A.; Ibragimov, A. G.; Dzhemilev, U. M. Russ. J. Org. Chem. 2013, 49, 1686-1689. http://dx.doi.org/10.1134/S1070428013110213

22. Makhmudiyarova, N. N.; Mudarisova, L. V.; Meshcheryakova, E. S.; Ibragimov, A. G.; Dzhemilev, U. M. Tetrahedron. 2015, 71, 259-265.

http://dx.doi.org/10.1016/j.tet.2014.11.064 